Br Heart $\mathcal{F} 1985$; 53: 349-52

\title{
Correspondence
}

\section{Lead specificity of the maximum ST/heart rate slope}

\section{response}

Sir,

We read with interest the paper by Balcon et al (1984; 52: 304-7) describing a relatively poor correlation between the maximum ST/heart rate slope and the severity of coronary artery disease at angiography. While this and most studies to date have been based on the derivation of this variable from conventional 12 lead electrocardiographic systems with an additional precordial electrode, CM5, we have recently obtained similar results with a computerised Frank orthogonal lead system. ${ }^{1}$ In 98 men studied by upright bicycle ergometry and coronary angiography, the maximum rate of change of ST amplitude was a poor predictor of the extent of coronary artery disease. Although a broad relation existed between the magnitude of this variable and the number of significantly diseased vessels, defined as $>\mathbf{7 5} \%$ luminal stenosis, the degree of overlap was such that accurate discrimination of vessel disease groups was not achieved.

Advocates of the use of the maximum ST/heart rate slope as an index of severity of coronary atherosclerosis suggest that the slope may be selected from any lead in which the linear regression of ST change on heart rate is significant. This assumes that the ST response to exercise is uniform in all leads and that a common pathophysiological mechanism-namely, a mismatch of myocardial oxygen supply and demand-is the sole determinant of such a response. Using the Frank lead system we have found that these assumptions are invalid and that responses are lead specific. $^{2}$ Maximum rates of change of ST amplitude are similar in leads $X$ and $Z$ but greater in lead Y. It is likely that this disparate response is due to a disproportionate effect of exercise induced augmentation of the atrial repolarisation ( $\mathrm{Ta}$ ) wave on the early part of the ST segment in vertically orientated leads. ${ }^{3}$

This non-uniformity in lead response is evident in the data presented by Balcon et al, who documented the lead from which the maximum ST/heart rate slope was sampled in 27 patients, 19 of whom had single vessel disease. This vessel disease group comprised eight patients with right coronary artery and 11 with left anterior descending disease. Although dominance of the coronary circulation is not reported, precluding estimation of the amount of jeopardised myocardium subtended in each patient, we may assume that this subpopulation is homogeneous, and as such max- imum ST/heart rate slopes should be comparable. It is clear, however, that slopes sampled from leads II, III, and $\mathrm{aVF}$, which are predominantly vertically orientated, tend to be of greater magnitude (mean 41) than those from lateral leads I, aVL, V4-6 (mean 31). Slopes selected from CM5 which has both vertical and horizontal components are even greater (mean 48) suggesting the influence of additional confounding variables.

The relative contributions of normal physiological responses and underlying pathology as determinants of the maximum ST/heart rate slope appear to be lead dependent, and the development of lead specific criteria for normal and abnormal ST/heart rate slope responses may improve diagnostic accuracy and facilitate the development of a multivariate model for better assessment of overall myocardial ischaemia.

J M Beattie,

G B Seibert,

C G Blomqvist,

Division of Cardiology,

University of Texas Health Science Center,

5323 Harry Hines Boulevard,

Dallas,

Texas 75235,

USA.

\section{References}

1 Beattie JM, Seibert GB, Wilson N, Pipberger HA, Blomqvist CG. Clinical assessment of the maximum rate of change of ST amplitude derived from a computerized orthogonal exercise ECG system. In: d'Alche P, ed. Proceedings of the 11th international congress on electrocardiology. 1985 (in press).

2 Beattie JM, Seibert GB, Wilson N, Pipberger HA, Blomqvist CG. Determinants of the normal maximal ST/heart rate slope in a computerized orthogonal lead system. In: Ripley KL, Ostrow HG, eds. Computers in cardiology. Silver Springs: IEEE Computer Society Press, 1985 (in press).

3 Lepeschkin E. Physiological factors influencing the electrocardiographic response to exercise. In: Blackburn $\mathrm{H}$, ed. Measurement in exercise electrocardiography. The Ernst Simonson Conference, Minneapolis 1967. Springfield, Illinois: Thomas, 1969:363-87. 